\title{
Alternance stylistique et cohésion intra-groupe dans une banlieue multiethnique de Paris
}

\author{
Zsuzsanna Fagyal \& Christopher Stewart \\ Département de Français \\ Université de l'Illinois à Urbana-Champaign \\ \{zsfagyal ; cmstewrt\}@uiuc.edu
}

\section{Introduction}

Pendant longtemps, la branche de la sociolinguistique communément appelée la linguistique de contact 'contact linguistics' semblait s'intéresser peu au transfert des traits prosodiques ${ }^{1}$ dans des situations de multilinguisme. Dans un compte-rendu récent consacré au contact en situations d'immigration, M. Clyne (2003:71) qualifia cet état des choses de «paralytique», alors que d'autres parlaient de la marginalisation de la prosodie au profit d'autres phénomènes phonétiques et morpho-syntaxiques (Cruz-Ferreira 1999). Mais les dix dernières années semblent avoir apporté de grands changements, car les études portant sur l'intonation en contact se sont multipliées en anglais. Bien qu'elles soient parfois très différentes dans leurs approches à l'intonation et à la problématique du contact entre les langues, elles montrent sans équivoque que, tout comme d'autres phénomènes phonologiques, les phénomènes tonals peuvent être empruntés ('borrowing') et mélangés ('mixing') dans la parole des locuteurs bilingues.

On trouve, entre autres, qu'au Pérou les accents intonatifs ('pitch accents') montants sont réalisés plus tôt dans les syllabes accentuées prononcées par des locuteurs bilingues espagnol-quechua que dans celles des locuteurs hispanophones unilingues dans la même communauté (O'Rourke 2004). Dans une situation de passage ('shift') de la langue locale de l'île d'Okinawa au japonais, on montre également que les accents de mots distincts de la langue locale moribonde sont en train de se transférer dans la prosodie des mots japonais parlés par les locuteurs unilingues et démographiquement dominants de l'île (Heffernan 2006). Dans un contexte européen, Simonet (2008) trouve que les populations hispanophones établies sur l'île catalane de Majorca ont tendance à formuler leurs questions totales avec une intonation descendante, empruntant la forme mélodique des interrogatives attestées en catalan.

De nombreuses études suggèrent que l'Europe occidentale constitue un terrain particulièrement propice à l'observation du contact entre les langues. La parole des jeunes adultes et des enfants multilingues issus de l'immigration de la main-d'œuvre d'origine non-européenne après la Seconde Guerre mondiale semble contenir de nombreux phénomènes prosodiques pragmatiquement marqués et perçue comme nonstandard par la société unilingue. De tels traits ont été relevés notamment en Angleterre, en Suède, en Allemagne, et en France.

Gumperz $(1978,1982)$ était le premier à observer que l'anglais britannique des immigrés indiens et pakistanais témoigne de nombreuses interférences avec les langues d'origine. Ces traits, dont des phrases intonatives courtes frappées par un accent final, semblaient être évalués négativement par les Britanniques. Plus récemment, les patrons intonatifs "non-suédois", caractérisés par "un ton montant inattendu et un rythme inégal" (Kotsinas 1998:136), ont été attestés dans la parole des adolescents suédois de Rinkeby, une banlieue populaire de Stockholm. Ces intonations constitueraient désormais des indices permettant aux Suédois de distinguer perceptuellement les habitants des quartiers de Rinkeby des « habitants ordinaires de Stockholm» (Kotsinas 1998, 2001). La parole des enfants bilingues allemands d'origine turque témoigne également de plusieurs contours intonatifs de fin de phrases prosodiques issus du contact entre les deux langues (Queen 1996). Ces études démontrent que les traits prosodiques issus du contact entre les langues peuvent constituer des indices ${ }^{2} d^{\prime}$ 'appartenance symbolique dans une communauté bilingue. 
De tels cas ont également été identifiés dans le français populaire en contact avec les langues de l'immigration (Gadet 1998, Conein et Gadet 1998). En plus du lexique non-standard très saillant, des contours intonatifs frappant par leur nouveauté ont été identifiés dans les parlers de certains quartiers et de groupes issus de l'immigration:

\begin{abstract}
«C'est donc avant tout dans le lexique et la prosodie que l'on peut situer une réelle spécificité de cette nouvelle langue populaire. [...] Si le lexique constitue de tout temps une dimension mouvante de la langue, l'observation de la prosodie permet de faire une remarque véritablement instructive. » (Gadet, $1998: 22$ ).
\end{abstract}

Similaires à des contours emphatiques attestés en français de référence (Di Cristo 1998), ces intonations semblent être marquées d'un point de vue pragmatique (Fagyal 2005, Stewart et Fagyal 2005, Le Gac et al. 2006) : « une première écoute produit immédiatement un effet d'étrangeté frappant » (Conein et Gadet 1998:108). Dans son travail de thèse portant sur la prosodie des jeunes d'une banlieue rouannaise, I. Lehka-Lemarchand (2007:345) qualifie de tels indices mélodiques marqués d'un "phénomène fortement stigmatisé et stigmatisant $»$.

\title{
2 Problématique et hypothèses
}

A la lumière de ces observations, l'intuition de Gumperz semble confirmée: certains traits prosodiques représentent des indices d'ethnicité, et ceci dans plusieurs communautés européennes urbaines. Néanmoins, il existe à l'heure actuelle peu d'études montrant que la présence des indices prosodiques d'appartenance ethnique est soumise à des variations stylistiques dont les mécanismes sont encore mal connus. La plupart des études préalables restreignent leurs champs de vision aux contours marqués dans la parole des locuteurs bilingues ou se tournent vers les productions médiatisées.

R. Queen (2004:516-517), par exemple, montre que dans les films américains doublés en allemand, les passages prononcés en anglais américain noir vernaculaire sont rendus en allemand par des traits phoniques caractéristiques des parlers urbains populaires et de la parole des jeunes issus de l'immigration turque. La mise en scène cinématographique des accents permet donc d'observer les moyens langagiers par lesquels «un caractère est mis en place rapidement». A travers l'usage qu'en fait le cinéma, la variabilité linguistique d'un parler peut donc être stylisée au point où on fait appel à «des styles préexistants encore plus stéréotypiques que les variations dans la vie sociale réelle ». Reste-t-il encore à savoir comment cette variation stylistique est structurée dans les interactions réelles.

Par la suite, nous présenterons une étude de cas centrée sur un groupe d'adolescents enregistrés lors d'une enquête de terrain dans une banlieue populaire de Paris. Nous analyserons les contours intonatifs relevés en présence de l'enquêtrice et des amis du locuteur présents lors de l'enregistrement. Nous illustrerons des instances d'accommodation et d'alignement du locuteur avec les différents membres de son groupe d'amis, son audience, à travers l'usage de moyens prosodiques. Nous proposerons d'interpréter ces instances comme des alternances stylistiques dans le cadre du modèle du « style pour l'audience » ('style as audience design') de A. Bell (1984, 2001). Bien que non-exhaustive, l'analyse illustrera des phénomènes prosodiques authentiques émergeant dans des contextes d'interactions réelles. Elle permettra donc de situer les formes mélodiques marquées et attestées dans plusieurs études préalables.

\section{Corpus et méthode}

Le corpus provient des enregistrements réalisés lors d'une enquête de terrain effectuée entre 2000 et 2002 dans un collège d'une banlieue populaire de Paris. Les adolescents interviewés étaient nés en France de parents d'origine africaine ou d'autres pays non européens. Les extraits étudiés correspondaient à des commentaires d'images visant la répétition de certains mots-cibles destinés à fournir un échantillon représentatif de voyelles orales. Cette technique connue dans les études phonologique dites «de laboratoire » était nécessaire pour provoquer la répétition de mots-cibles identiques dans les mêmes contextes discursifs chez tous les locuteurs. Les commentaires d'images, plus spontanés que la lecture à 
voix haute, étaient donc préférés à la lecture de textes ou de listes de mots préconisés par le paradigme labovien de terrain.

La tâche des locuteurs consistait d'abord à identifier ce qu'ils voyaient sur chaque image, et ensuite à décrire ces images en détail. Ensuite, une ou deux questions visant à obtenir leur opinion sur les images étaient posées. Cette tâche était présentée aux adolescents comme un jeu d'associations libres.

Les images étaient également destinées à maintenir l'intérêt des adolescents. Par exemple, une série de photos représentant des célébrités, des joueurs de football, ainsi que des photos et des dessins représentant des activités et des scènes liées à la vie quotidienne des adolescents étaient proposées. Ces images étaient alternées avec des dessins représentant certaines lettres de l'alphabet, visant principalement à obtenir la répétition des voyelles en isolation. ${ }^{3}$ Sur une soixantaine de mots-cibles présentés sur trente-quatre images, plusieurs mots se trouvaient en position finale de phrase intonative, la principale unité prosodique en français, ce qui nous a permis d'effectuer les premières comparaisons entre les locuteurs. Par la suite, nous présenterons les résultats d'une première analyse quantitative cumulée, suivie de trois instances d'alternance stylistique issues de deux extraits de parole.

\section{Exemples d'alternance stylistique}

\subsection{Le comptage des occurrences}

Afin d'avoir une idée de la variété des formes intonatives utilisées par les locuteurs lors de l'énumération des objets et des individus représentés sur les images, nous avons étiqueté chacun des mots-cibles en fonction du type de contour intonatif observé. La Figure 1 montre le résultat du comptage de trois types de contour chez les deux locuteurs : Yasin Z et Ismail N, tous les deux adolescents de 14 ans, né en France des parents d'origine algérienne et d'origine africaine équatoriale, respectivement. En plus du français, les deux parlent arabe et, selon les mots d'Ismail, «malien » à la maison.

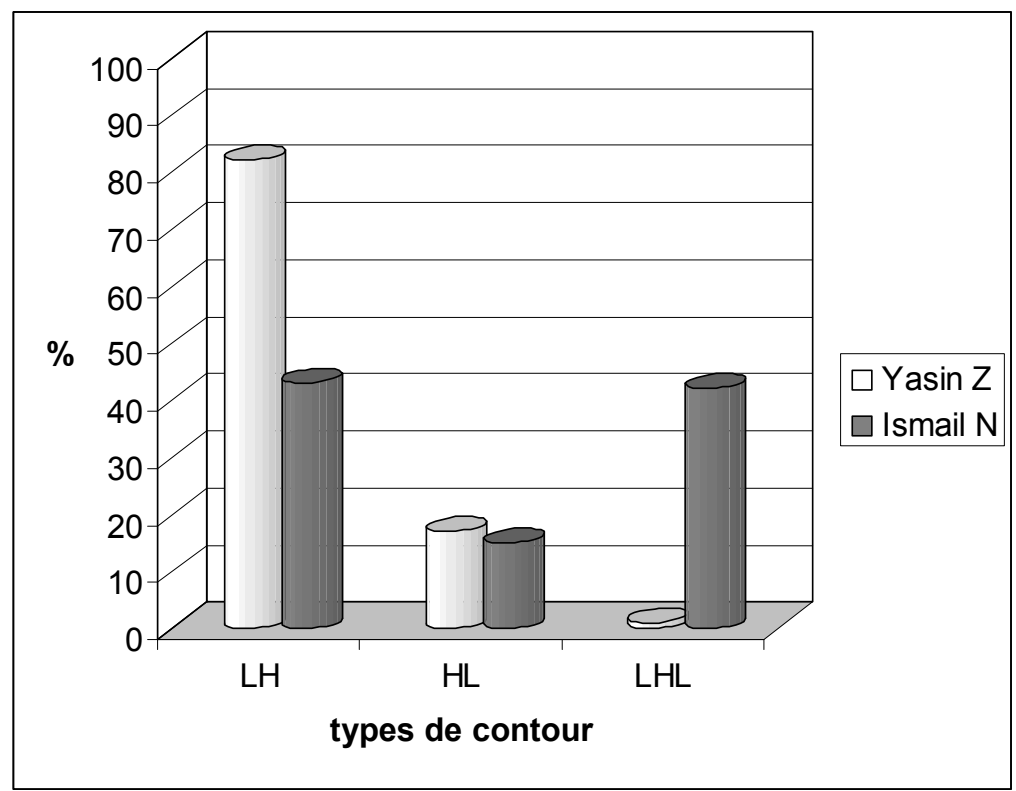

Figure 1. Nombre de réalisations de contours montant 'continuatifs' (LH), descendant (HL) et montantdescendant (LHL) en position finale accentuée de soixante-deux mots-cibles. 
Comme en témoigne la Figure 1, l'écrasante majorité des soixante-deux mots-cibles recensés (voir Annexe ci-dessous) ont été prononcées par les deux locuteurs avec un contour intonatif montant sur la dernière syllabe du mot-cible. Ces contours sont attestés et largement reconnus comme « des montées de continuation », c'est-à-dire des formes canoniques délimitant la frontière droite du syntagme accentuel dans le français dit «de référence » (voir par exemple Di Cristo 1998). Une partie mineure des réalisations étaient descendantes en syllabes toniques finales de mot, phénomène également constaté dans les contextes d'énumérations en français (Guaïtella 1991). Ces deux formes mélodiques ne semblaient donc représenter aucun usage marqué du français dit «de référence ». Les cas où ni de montée ni de descente intonative marquée ont pu être décelées, le contour était classifié comme descendant. Un petit nombre de réalisations dans la parole de Yasin $\mathrm{Z}$ et une quantité plus importante chez Ismail $\mathrm{N}$ avaient, en revanche, des caractéristiques mélodiques différentes de ces deux formes canoniques. Ces contours montants-descendants affichaient une montée dans la pénultième suivie d'une chute brusque sur la syllabe finale du mot. Selon de nombreuses études, il s'agirait d'un des phénomènes prosodiques stéréotypiquement associé avec l'accent dit «des banlieues » (Conein et Gadet 1998, Fagyal 2003a, b, 2005, Le Gac et al. 2006).

Dans le cas de Yasin, les montées de continuation $(\mathrm{LH})$ « de référence » prédominent, alors que dans les énumérations des mots-cibles par Ismail les contours LH sont numériquement aussi importants que les contours (LHL) qui sont pragmatiquement marqués. Comme la comparaison qualitative le révélera par la suite, la différence principale entre les deux locuteurs ne réside pas uniquement dans les proportions d'usage du contour dans les deux contextes. Le contexte - ou style contextuel selon le paradigme labovien - représente une dimension importante, mais qui est loin d'être unique. La différence entre les deux locuteurs réside également dans la contextualisation du contour marqué. Alors qu'Ismail prononce une bonne partie des mots-cibles avec cette intonation marquée, Yasin utilise le contour LHL uniquement en dehors du contexte du commentaire des images. Il convient donc d'examiner chacun de ces contextes d'usage.

\subsection{Alternance stylistique initiative}

Le premier cas d'alternance stylistique est celui de Yasin. Lors de son enregistrement, Yasin utilisait systématiquement des contours intonatifs montants sur des mots cibles en fin de phrase intonative lorsqu'il adressait ses propos à l'enquêtrice. La Figure 2 illustre un contour de ce type.

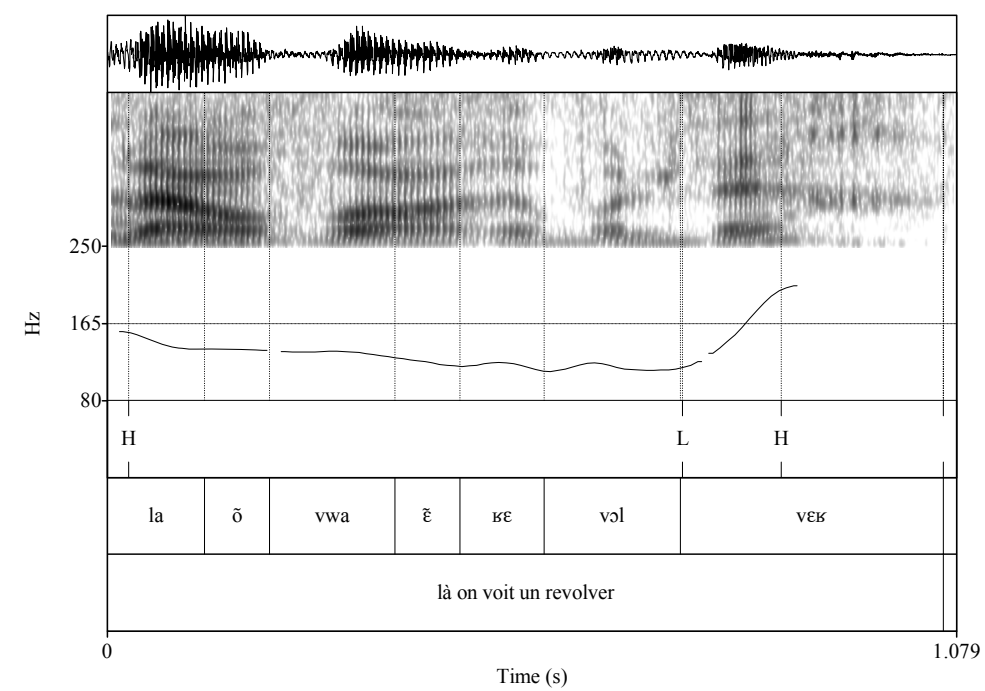

Figure 2. Là, on voit un revolver, énoncé prononcé avec un contour intonatif montant sur le mot cible revolver. Ce patron intonatif est bien connu comme 'contour continuatif' dans les descriptions de l'intonation du français de référence (Di Cristo 1998). 
La courbe mélodique (de fréquence fondamentale ou $f 0$ ) affichée sur une plage de variations de $80 \mathrm{~Hz}$ à $250 \mathrm{~Hz}$ au milieu de la figure montre une montée marquée sur la dernière syllabe du mot -ver, alors que les syllabes précédentes sont prononcées sur un ton bas. Plusieurs prises de parole illustrant les occurrences de tels contours sont transcrites dans Extrait (1) ci-dessous.

L'échange entre Yasin et l'enquêtrice s'est déroulé devant un public d'amis et de camarades de classe relativement actif. L'enregistrement ayant eu lieu à l'intérieur du collège dans une salle de classe vide mais accessible aux élèves pendant les récréations, il y avait parfois plusieurs élèves intéressés qui assistaient aux enregistrements. Ceci a permis de faire plusieurs observations sur la dynamique des échanges dans les groupes des paires. Certains auditeurs tenaient, par exemple, à participer de manière active dans l'interaction, alors que d'autres ne paraissaient que des figurants silencieux. L'un des auditeurs les plus actifs était Ismail, le deuxième locuteur analysé ici, qui semblait adopter le rôle de «l'animateur principal » dans le groupe constitué spontanément autour de Yasin. Par conséquent, l'enregistrement de celui-ci s'est rapidement transformé en un véritable spectacle devant ses pairs. Dans Extrait (1), Ismail et l'un de ses meilleurs amis font des remarques sur Yasin et les images dont il faisait les commentaires.

\section{Extrait (1)}

$\begin{array}{lll}1 & \text { YasinZ } & \text { Là on voit un revolver }(\boldsymbol{L H}) \text { Ça doit être un Magnum } 357 \text { piton }(\boldsymbol{L H}) \\ 2 & \text { E \& audience } & \text { Ouais. } \\ 3 & \mathrm{E} & \text { Ça t' fait penser à quoi ? } \\ 4 & \text { YasinZ } & \text { Ça m' fait penser à, euh, à une arme }(\boldsymbol{L H}) \\ 5 & \mathrm{E} & \text { Bon ben, c'est une arme (pause). Vas-y, continue ! } \\ 6 & \text { Ami n }{ }^{\circ} 1 & \text { Comme Lucky Luc. } \\ 7 & \text { IsmailN } & \text { [Attends, je veux l' voir !] } \\ 8 & \text { Ami n }{ }^{\circ} 2 & \text { [Moi aussi.] } \\ 9 & \text { YasinZ } & \text { Là on voit une voiture }(\boldsymbol{L H}) \quad \text { (pause) Là on voit à l'arrière il y a un pneu }\end{array}$

Quelques minutes après cet échange, les mêmes amis se disputaient avec l'enquêtrice à propos de leur accès au microphone, empêchant Yasin de continuer son enregistrement. Celui-ci, finissant par en avoir assez de ne pas pouvoir continuer, il «change de style» au sens prosodique et lexical du terme. Au moment où, à cause des chevauchements incessants des voix, il n'arrive plus à parler, il sort de son rôle de sujet d'expérience, et il fait taire Ismail de façon bien plus efficace que ne le fait l'enquêtrice. Il semblerait qu'il parvienne à remettre son camarade en place — et à produire un silence immédiat autour de lui - par une alternance de style brusque et inattendu. Au lieu de continuer sur un ton neutre qu'il employait tout au long de l'échange avec l'enquêtrice, Yasin a recours au lexique non standard et au contour intonatif LHL associé avec l'accent dit «des banlieues». Un exemple de ce contour, tiré de l'échange même est représenté sur la Figure 2.

Dans la taxonomie des interactions déclenchant des alternances stylistiques, Bell (2001) et Coupland (2007) proposent de distinguer entre alternance stylistique initiative et réactive. La première catégorie confère un rôle actif au locuteur principal de l'échange qui, lorsqu'il le voit utile et nécessaire, peut signaler une attitude (stance) différente envers son audience par un changement de style. Celui-ci peut se manifester par différents moyens langagiers. Il nous semble que cet exemple — dans la mesure où il se 
retrouve de façon consistante dans le reste des interactions avec les enfants - constitue une bonne illustration de ce type d'usage du style à des fins interactionnelles.

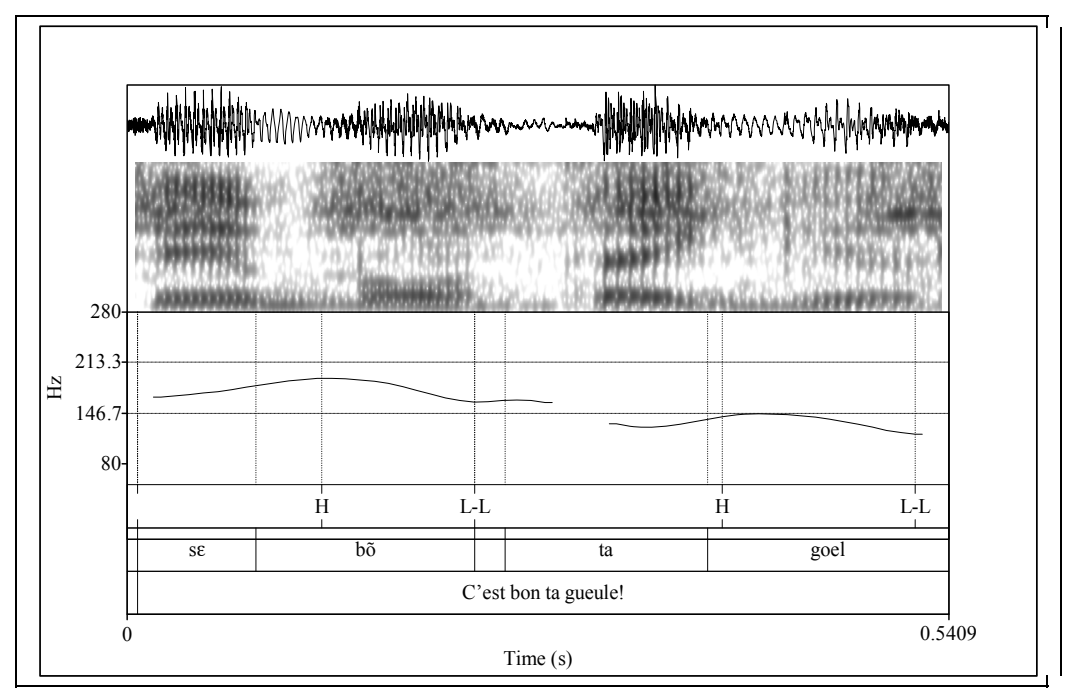

Figure 3. C'est bon. Ta gueule! Propos de Yasin adressée à l'un de ses pairs l'empêchant de continuer son enregistrement. La forme intonative des deux énoncés prend la forme de contours du type LHL en fin de phrase, intonation associée à l'accent des banlieues.

\subsection{Alternance stylistique réactive}

L'Extrait 2 était relevé lors d'un échange similaire à l'extrait précédent. Cette fois-ci, en revanche, Ismail et son meilleur ami et camarade de classe, Basil B, un adolescent de 14 ans né en France de parents non issue de l'immigration, font conjointement le commentaire des images présentés à Ismail. «La collaboration » entre amis se manifeste sous différents aspects. L'un des aspects est la co-construction de l'énonciation, dont les mécanismes ont été analysés en détail, entre autres, par Jeanneret (1999) dans la parole médiatisée en français. Comme le montrent les lignes 10 et 13 dans l'Extrait 2, ainsi que les illustrations de contours mélodiques placés sous les mots-cibles revolver, Ismail $\mathrm{N}$ et son ami semblent chercher un consensus commun sur le mot référant à l'image qui leur est présentée.

La première fois où le mot-cible revolver apparaît dans la conversation (ligne 3), il est énoncé avec une intonation montante du type LH, typique à d'autres contours d'énumération, mais après une première tentative de nommer cet objet pistolet (ligne 10). Alors que l'enquêtrice cherche à poser une question (ligne 5) à Ismail, Basil intervient (ligne 4) simultanément. Il y a donc chevauchement des voix lors duquel le même mot est répété avec une intonation descendante (non illustrée ici). Aussitôt après, Ismail manifeste à son ami son acceptance de ce terme, et il le répète à nouveau (ligne 6). Cette fois-ci, en revanche, Ismail prononce le mot plus fort, à un rythme plus rapide et surtout avec une intonation marquée du type LHL qu'il utilise bien plus fréquemment lors de l'énumération des mots-cibles et la description des images que Yasin (voir ci-dessus).

Selon le modèle de «style pour audience» de Bell (2001), ce type d'alternance stylistique peut être interpréter comme une instance d'alternance stylistique 'réactif'. Dans ces termes-là, l'adoption par Ismail du contour intonatif de son ami correspondrait à un signe inconscient d'alignement immédiat avec le comportement langagier de son ami et interlocuteur. 


\section{Extrait (2)}

$1 \quad \mathrm{E}$

quoi...]

2 BasilB

3 IsmailN
OK. Donc même chose : tu peux nous dire l'objet, le nom de l'objet [et à

[C'est un pistolet 350. Ha ha !]

[Pisto.. euh...] Revolver (LH)

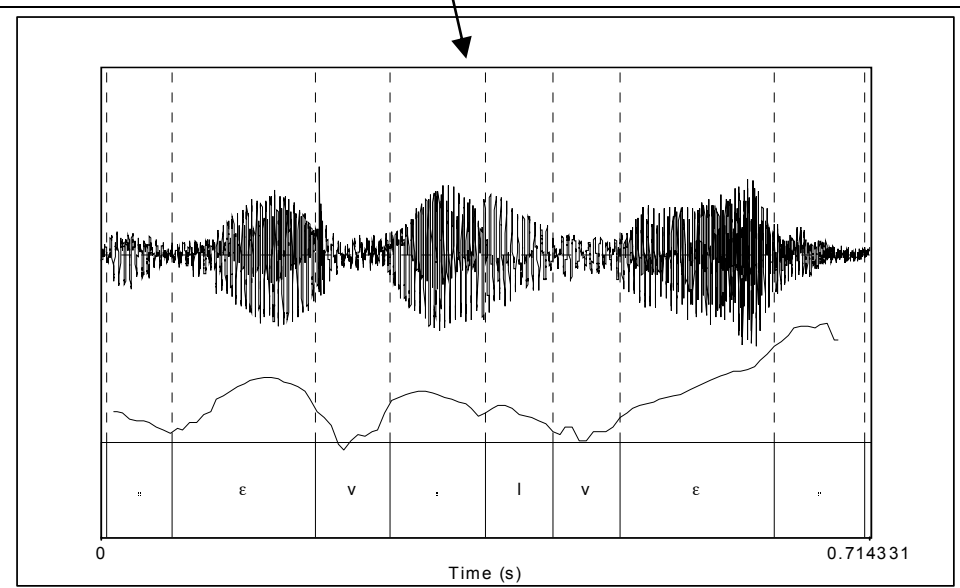

$4 \quad$ BasilB

Un révolver. [Ah oui, ah oui!]

$5 \quad \mathrm{E}$

[Ca te rappelle...]

$6 \quad$ IsmailN

Revolver $(\boldsymbol{H L})$

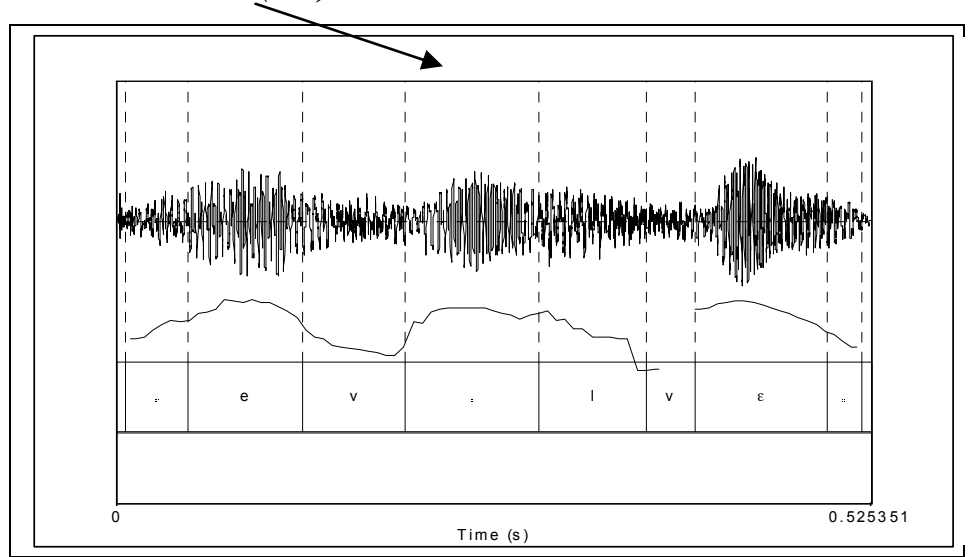

$7 \quad \mathrm{E}$

[Est-ce qu'une idée...]

$8 \quad$ BasilB

Revolver

(pause) 


\section{Conclusion et discussion}

Dans cet article, nous avons cherché à illustrer, dans la parole de deux adolescents enregistrés dans un contexte d'énumération et utilisant surtout une prosodie typique au français de référence, l'alternance entre plusieurs contours pragmatiquement marqués et non marqués dans des buts interactionnels précis. Dans les cas illustrés ci-dessus, il s'agirait d'une volonté de s'aligner avec ses pairs, et donc d'utiliser un système de signalisation affective - un type d'intonation - propre au groupe. L'un des locuteurs analysés semble se solidariser avec son ami participant à l'interaction en répétant le mot en question avec une intonation typique, ou du moins typiquement associée, à l'accent dit « des banlieues ». Dans le cas de l'autre locuteur, le contour typique au groupe des pairs permet de sortir du rôle du locuteur effectuant une tâche expérimentale pour communiquer un message personnel au groupe.

A travers ces analyses, on montre que la prosodie fait partie des outils linguistiques dont les locuteurs se servent pour modifier leur parole en fonction des participants présents dans l'interaction. Avec l'enquêtrice et un public ('audience') plus ou moins actif d'amis et de pairs, les locuteurs négocient constamment leur attitude ('stance') à travers l'usage de plusieurs contours. De tels usages des contours intonatifs montant et surnommés « uptalk» ont été attestés dans la parole des adolescentes texanes en anglais américain (McLemore 1991).

Le modèle théorique adapté dans cette étude est celui du « style pour audience » de A. Bell (1984, 2001), mettant l'emphase sur le rôle de l'audience participant de façon active à l'interaction. Conformément à l'interprétation de la notion du style par Coupland (2007: 58), les exemples ci-dessus ont confirmé que le style représente une dimension cruciale de l'interaction, et qu'il est « co-construit à travers l'engagement entre paires ou parties dans le discours ». Dans les extraits présentés, le besoin de la part des locuteurs de conserver leur réputation aux yeux de leurs pairs a pu servir de motivation principale pour leur alignement et leur ré-alignement avec le répertoire tonal de leurs paires, et donc pour l'emploi d'une prosodie non-standard à certains moments de l'interaction.

Contrairement à Coupland (2007) qui prend une position opposée aux approches quantitatives variationnistes, nous soutenons qu'il est important d'envisager l'étude du style dans ses aspects à la fois quantitatifs et qualitatifs. Le style étant un phénomène relationnel complexe par excellence, son étude nécessite la mise en œuvre des tous les moyens d'analyse dont on possède.

\section{Annexe}

\section{Mots cibles dont les mots plurisyllabiques ont servi dans le comptage du nombre d'occurrences des trois contours étudiés}

(Zinadine) Zidane, foot(ball), joueur de foot(ball), (Michael) Jordan, Tiger Woods, joueur de basket(ball), joueur de golf, golfeur, cités, joueurs de baskets, des noirs (des Reunois), les Etats-Unis, des boxeurs, Etats-Unis ou la France pistolet, revolver, gun, voiture, (avec un) pneu crevé, la lettre Vé, homme préhistorique avec sa massue, son bâton, homme de Cromagnon habillé en peau, a, bé, cé, les lettres de l'alphabet et majuscule et minuscule, souris, rat, queue, collier, dame (femme), blonde, légumes, chou, chou-fleur, petit garçon, (fait ses) lacets, feu (de camps), pou (dans les cheveux sur un peigne ou une brosse), poule, bottes, boue, chantier, ouvrier, ferme, lémure, singe, queue (de zèbre), jouets (de bébé), 
nounours, train, ballon, cubes, la lettre Cé, chat, poil hérissé, peur, dos courbé, zoo, animaux, lion, girafe, hippopotame, singe, muguet, (le premier) mai, épée, sabre, pelle, seau, étoile de mer, la lettre Pé, quai, bagage, voyage, train, le Petit Chap(e)ron Rouge, le loup, la corbeille, une fée, un ange, baguette magique, faire un vœu, un dé, la lettre Bé, la raie, le perroquet, le chat qui boit son lait, des baies ou des fruits, mûres, dattes.

\section{Références bibliographiques}

Bell, A. (1984). Language style as audience design. Language in Society 13, 145-204.

Bell, A. (2001). Back in style: Reworking audience design. In Eckert P. and J. Rickford (eds.) Style and Sociolinguistic Variation. Cambridge and New York : Cambridge University Press, 139-169.

Clyne, M. (2003). Dynamics of language contact. English and immigrant languages. Cambridge: Cambridge University Press.

Conein, B., \& Gadet, F. (1998). Le "français populaire" des jeunes de la banlieue parisienne entre permanence et innovation. In J. Androutsopoulos, K. \& A. Scholz (Eds.), Jugendsprache / Langue des jeunes / Youth language. Frankfurt: Peter Lang, 105-123.

Coupland, N. (2007). Style. Language Variation and Identity. Cambridge and New York: Cambridge University Press.

Cruz-Ferreira, M. (1999). Prosodic mixes: strategies in multilingual language acquisition. The International Journal of Bilingualism 3(1): 1-21.

Di Cristo, A. (1998). Intonation in French. In A. Di Cristo \& D. Hirst (Eds.), Intonation systems: a survey of twenty languages. Cambridge, New York: Cambridge University Press, 195-218.

Fagyal, Z. (2003a). La prosodie du français populaire des jeunes à Paris: traits héréditaires et novateurs. Le Français aujourd'hui, 143 (nº spécial "Français de l'école et langues des élèves : quel statut, quelles pratiques ?"), 47-55.

Fagyal, Z. (2003b). The Matter with the Penultimate: Prosodic Change in the Vernacular of Lower-Class Immigrant Youth in Paris, Proceedings of the XVth International Congress of Phonetic Sciences, Barcelona, 671-674.

Fagyal, Z. (2005). "Prosodic consequences of being a Beur: French in contact with immigrant languages in Paris". Selected papers from NWAV 32, Philadelphia, 2004, Working Papers in Linguistics 10 (2), 91-104.

Gadet, F. (1998). Des fortifs aux técis: persistance et discontinuités dans la langue populaire. In D. Marley, M.-A. Hintze, \& G. Parker (Eds.), Linguistic Identities and Policies in France and the French-Speaking World. London: Centre for Information and Language Teaching Research, 11-26

Guaïtella I. (1991). Rythme et parole: comparaison critique du rythme de la lecture oralisée et de la parole spontanée, Thèse de Doctorat, Université de Provence.

Gumperz, J., J. (1978). The Conversational Analysis of Interethnic Communication. In Ross, E. L. (ed.), Interethnic Communication. Athens: The University of Georgia Press, 13-31.

Gumperz, J., J. (1982). Discourse strategies. Cambridge: Cambridge University Press.

Heffernan, K. (2006). Prosodic levelling during language shift: Okinawan approximations of Japanese pitch accents. Journal of Sociolinguistics, 10 (5), 641-666.

Jeanneret, T. (1999). La coénonciation en français: Approches discursives, conversationnelle et syntaxique. Peter Lang, Bern, Berlin, Francfort, New York.

Kotsinas, U.-B. (1998). Language contact in Rinkeby, an immigrant suburb. In Androutsopoulos, K. J. and A. Scholz (Eds.), Jugendsprache / langue des jeunes / youth language. Frankfurt, New York, Paris: Peter Lang, 136-145.

Kotsinas, U.-B. (2001). Pidginization, creolization and creoloids in Stockholm, Sweden. In N. Smith \& T. Veenstra (Eds.), Creolization and Contact. Amsterdam, Philadelphia: John Benjamins, 125-155.

Labov, W. (1972). Sociolinguistic Patterns. Philadelphia: University of Pennsylvania Press.

Labov, W. (1994). Principles of Linguistic Change: Internal Factors. Oxford, Blackwell. 
Le Gac, D., Jamin, M., et Lehka, I. (2006). A preliminary study of prosodic patterns in two varieties of suburban youth speech in France, Proceedings of the Third International Conference on Speech Prosody, Dresden, 241244

Lehka-Lemarchand I. (2008). Accent de banlieue: Approche phonétique et sociolinguistique de la prosodie des jeunes d'une banlieue rouennaise. Thèse de doctorat de l'Université de Rouen.

McLemore, C. (1991). The Pragmatic Interpretation of English Intonation: Sorority Speech. Department of Linguistics, University of Texas in Austin.

O'Rourke, E. (2004). "Peak placement in two regional varieties of Peruvian Spanish intonation." Contemporary Approaches to Romance Linguistics. Eds. Auger, J., Clements, C. and Vance, B. Amsterdam: John Benjamins Publishing Company, 321-341.

Queen, M., R. (1996). Intonation in contact: a study of Turkish-German bilingual intonation patterns. Ph.D., University of Texas at Austin.

Queen, M. R. (2004). Du hast jar keene Ahnung: Dubbing African-American English into German. Journal of Sociolinguistics, 8(4), 517-537.

Simonet, M. (2008). Language contact in Majorca: An Experimental Sociophonetic Approach. Doctoral dissertation. University of Illinois at Urbana-Champaign.

Stewart, C., et Fagyal, Z. (2005). Engueulade ou énumération: Attitudes envers quelques énoncés enregistrés dans "les banlieues". In M.-M. Bertucci and Houdart-Merot, V. (eds.) Situations de banlieue: Enseignement, langues, culture. Paris: Institut National de Recherche, 241-252.

\footnotetext{
${ }^{1}$ Le terme prosodie englobe les variations de la fréquence fondamentale, de la durée, et de l'intensité, et certains aspects du timbre de la voix.

${ }^{2}$ Les notions d'indice, de marqueur et de stéréotype sont utilisées ici dans le sens défini par l'école variationniste : elles renvoient aux degrés différents d'investissement symbolique d'une communauté dans les variations linguistiques en cours (Labov 1994 : 300-301).

${ }^{3}$ Certaines images servaient pour de futures expériences.

${ }^{4}$ L'abréviation $\mathbf{E}$ réfèrera désormais systématiquement à 'enquêtrice'. Les contours seront indiqués par des lettres majuscules à côté des mots cibles et les chevauchements signalés en parenthèses crochets.
} 